\title{
Improved contamination control for a rapid phage- based rifampicin resistance test for Mycobacterium tuberculosis
}

\author{
Correspondence \\ Heidi Albert \\ heidialbert@finddiagnostics.org
}

Received 8 September 2006

Accepted 12 June 2007

\author{
Richard Mole, ${ }^{1} \dagger$ Andre Trollip, ${ }^{1}$ Celeste Abrahams, ${ }^{1} \ddagger$ Marlein Bosman ${ }^{2}$ \\ and Heidi Albert ${ }^{1} \S$
}

\author{
${ }^{1}$ Biotec Laboratories Ltd, Somerset Hospital, Greenpoint, Cape Town, South Africa \\ ${ }^{2}$ National Health Laboratory Service, Old City Hospital Complex, Greenpoint, Cape Town, \\ South Africa
}

\begin{abstract}
A prospective study was conducted of the rapid FASTPlaque-Response test for determination of rifampicin resistance in Mycobacterium tuberculosis with and without the addition of an antimicrobial supplement containing nystatin, oxacillin and aztreonam (NOA) to control specimen-related contamination. A total of 631 smear-positive sputum specimens was tested. The age of specimens ranged from 0 to 21 days. The NOA antimicrobial was effective at controlling contamination, with $4.1 \%$ of specimens contaminated when the NOA antimicrobial supplement was used compared with $13.9 \%$ contamination without NOA. Overall levels of interpretability of the test with NOA were $87.8 \%$ with specimens of $\leqslant 3$ days and $79.0 \%$ for all specimens. This compared with 70.1 and $73.8 \%$ readable results, respectively, from conventional culture-based drug susceptibility testing (DST). Sensitivity, specificity and overall accuracy of the FASTPlaque-Response test for rifampicin resistance were $98.1,96.3$ and $96.6 \%$, respectively, for all specimens with NOA, and 93.2, 96.3 and $95.9 \%$ without NOA, when compared with resolved conventional DST results. Inclusion of the NOA supplement reduced contamination, increased the number of interpretable results and did not adversely affect the performance of the FASTPlaque-Response test. Thus, the use of NOA improves the robustness of the test, facilitating its wider implementation.
\end{abstract}

\section{INTRODUCTION}

The emerging worldwide problem of drug resistance complicates tuberculosis (TB) control measures. Multidrug resistance (MDR), defined as resistance to at least rifampicin and isoniazid, is a particular problem in the treatment of TB, as these are the two most effective drugs used in short-course chemotherapy (WHO, 2004). Rifampicin resistance is an effective surrogate marker for MDR TB in many settings (WHO, 2003) and is a useful predictor of treatment failure (Suarez et al., 2002). Recent estimates predict that there were between 376000 and 620000 new MDR TB cases in 2004 (Zignol et al., 2006).

tPresent address: Nottingham University Business School, Jubilee Campus, Wollaton Road, Nottingham NG8 1BB, UK.

$\ddagger$ Present address: Medical Biosciences Department, University of the Western Cape, Bellville, South Africa.

§Present address: Foundation for Innovative New Diagnostics (FIND), Cape Town, South Africa.

Abbreviations: DST, drug susceptibility testing; MDR, multi-drug resistance; NHLS, National Health Laboratory Service; NOA, nystatin, oxacillin and aztreonam; TB, tuberculosis.
Conventional methods of detecting TB drug resistance rely on culture-based drug susceptibility testing (DST). In solid culture systems, this can take up to several months. This period can be reduced to 3-4 weeks by the use of newer liquid-based culture systems (Heifets \& Cangelosi, 1999). New rapid methods of DST directly from clinical specimens will enable earlier detection of resistance, which is expected to improve treatment outcomes and decrease transmission (Fischl et al., 1992).

Several new rapid DST methods have emerged recently (Pai et al., 2006). These include phage-based methods (Jacobs et al., 1993; Wilson et al., 1997) and DNA amplification/ hybridization technologies (Morgan et al., 2005). These methods can be applied directly to smear-positive sputum specimens. One of these methods, the FASTPlaque technique, uses bacteriophage to report the presence of viable Mycobacterium tuberculosis in a specimen (Seaman et al., 2003; Wilson, 1997). The technique has been applied to both the detection of $M$. tuberculosis from clinical specimens (Albert et al., 2002; Muzaffar et al., 2002) and DST of M. tuberculosis cultures (Albert et al., 2001; Kisa et al., 2003; Wilson et al., 1997). Recently, it has also been applied to DST directly from smear-positive sputum 
(Albert et al., 2004), now commercialized as the FASTPlaque-Response test. Portions of decontaminated sputum specimen are incubated in the presence and absence of rifampicin, and the occurrence of plaques (clear zones) reflects the viability of $M$. tuberculosis after incubation with the drug, as bacteriophage require viable M. tuberculosis to replicate and lead to plaque formation. However, the proportion of uninterpretable results, either due to overgrowth of contaminating micro-organisms present in sputum or due to insufficient plaques in the control (drug-free) plate, has been identified as a potential limitation to the test's use.

This study evaluated the use of an antimicrobial supplement containing nystatin, oxacillin and aztreonam (NOA) to control contamination in the FASTPlaque-Response test and thus increase the proportion of interpretable results. In addition, the effect of the NOA antimicrobial supplement on the performance of the test was determined. The effect of specimen storage times on test performance was also evaluated.

\section{METHODS}

Specimens. From 1 March to 10 May 2005, smear-positive sputum specimens were collected in the National Health Laboratory Service (NHLS) laboratory, a large South African TB referral laboratory. Transport of specimens from clinics to the laboratory was at ambient temperature, with refrigerated overnight storage at the laboratory prior to processing. Specimens received on Friday were stored over the weekend at $2-8{ }^{\circ} \mathrm{C}$.

Smear-positive sputum sediments (1+ or greater; Enarson et al., 2000) of $0.5 \mathrm{ml}$ or larger were included in this study. Specimens were collected consecutively, according to the selection criteria, from those available in the NHLS laboratory, up to a total of approximately 20 samples per day.

Sample processing. Specimens were decontaminated using the NALC-NaOH procedure (Kent \& Kubica, 1985) within the NHLS laboratory. Following suspension of the pellet in phosphate buffer ( $\mathrm{pH} 6.8), 0.1 \mathrm{ml}$ was removed for a smear, and approximately $0.5 \mathrm{ml}$ was inoculated into MGIT culture (BD Biosciences). The remainder of the pellet (approximately $0.5 \mathrm{ml}$ ) was stored at room temperature for approximately $4 \mathrm{~h}$ until smear results were available and selection of smear-positive specimens could be made.

Smear microscopy. Smears of sediments were prepared, stained with auramine $\mathrm{O}$ and analysed by NHLS staff. Smear results were classified according to International Union Against Tuberculosis and Lung Disease guidelines (Enarson et al., 2000).

MGIT-960 culture. All specimens were processed for cultivation using the MGIT-960 system according to the manufacturer's recommendations, including the use of PANTA antibiotic supplement (BD Biosciences). Positive cultures were confirmed by acid-fast staining, and cultures free of contamination underwent identification and susceptibility testing.

Culture confirmation and DST. Conventional DST was performed using a modified 7H11 proportion method (Kent \& Kubica, 1985), with final concentrations of $1 \mu \mathrm{g}$ rifampicin $\mathrm{ml}^{-1}$ and $0.2 \mu \mathrm{g}$ isoniazid $\mathrm{ml}^{-1}$. At the same time, isolates were confirmed to be
M. tuberculosis complex by lack of growth on Lowenstein-Jensen medium containing p-nitrobenzoic acid (Allen, 1984).

FASTPlaque-Response testing. NOA was added to FASTPlaqueResponse Medium Plus (Middlebrook 7H9-based medium containing oleic acid/albumin/glucose/catalase-based supplement) to give a final concentration of $50000 \mathrm{IU}$ nystatin $\mathrm{l}^{-1}, 2 \mathrm{mg}$ oxacillin $\mathrm{l}^{-1}$ and $30 \mathrm{mg}$ aztreonam $\mathrm{1}^{-1}$. Medium Plus, either with or without NOA, was then used throughout the test procedure for FASTPlaque-Response.

Sediments (from the decontaminated pellet) of between 0.5 and $1.0 \mathrm{ml}$ were made up to $1 \mathrm{ml}$ with phosphate buffer $(\mathrm{pH} \mathrm{6.8)}$ ) and homogenized using a vortex mixer. Two $0.5 \mathrm{ml}$ portions were removed from each sediment and processed by the FASTPlaqueResponse method, according to the manufacturer's instructions (Biotec Laboratories), in either the presence or absence of NOA.

Result plates from Saturday were stored at $2-8{ }^{\circ} \mathrm{C}$ and read on Monday morning. All other plates were read on the day immediately after the second overnight incubation. Interpretation of test results was according to the manufacturer's instructions. For each specimen, the drug-free (RIF-) sample had to contain 100 plaques or more to be considered valid for interpretation. The specimen was determined to be rifampicin-susceptible if there were $\leqslant 49$ plaques in the drugcontaining $(\mathrm{RIF}+)$ sample, or as rifampicin-resistant if $\geqslant 50$ plaques were observed.

rpoB mutation analysis. Identification of mutations in an $81 \mathrm{bp}$ region of the $r p o B$ gene encoding rifampicin resistance was performed as described by Victor et al. (1999) using a dot-blot hybridization method, and conventional DST was repeated on the isolated cultures of specimens in which the FASTPlaque-Response results and conventional DST disagreed.

\section{RESULTS AND DISCUSSION}

\section{Conventional testing results}

A total of 631 smear-positive sputum specimens $(1+$ or greater) was used in the study. The number of specimens that were $1+, 2+$, and $3+$ smear-positive was 148 $(23.5 \%), 108(17.1 \%)$ and $375(59.4 \%)$, respectively. Specimens were from new cases (8), new suspects (67), retreatment suspects (123) and retreatment cases (63). The remaining specimens (370) were from patients of unknown category (data not provided with specimen examination request forms).

A total of $3(0.6 \%)$ non-TB mycobacteria were identified from 486 isolated mycobacterial cultures, but no further work was performed to speciate these isolates. Positive MGIT cultures (containing M. tuberculosis complex) took an average of 14.5 days (range 5-56 days) to become positive. DST took a further 21 days to yield results. Hence, a total theoretical elapsed time for the culture-based determination of rifampicin resistance was an average of 35.5 days (26-77 days). In contrast, the FASTPlaqueResponse test yielded results in 2 days (or 4 days if plates were kept over the weekend to be read on the Monday) following initiation of testing.

MDR was seen in 56 (8.9\%) of the specimens. Rifampicin resistance without isoniazid resistance occurred in only 
four specimens $(0.6 \%)$. Isoniazid resistance without rifampicin resistance occurred in 83 specimens $(13.2 \%)$. A high correlation $(92.9 \%)$ of rifampicin resistance to MDR TB was found in this population.

In this study, out of a total of 631 samples, 238 specimens were $\leqslant 3$ days old, whilst 391 specimens were $\geqslant 4$ days prior to processing. Two specimens did not have a collection date provided and were not included in further analyses. Almost half (190) of these older specimens were 4 days old and were due mainly to storage over the weekend prior to testing on a Monday. In addition, the occurrence of older specimens was due to their collection from outlying centres and their subsequent delay in transportation to the central testing facility in Cape Town. Specimens up to 21 days old were encountered and included in the study.

\section{Interpretability of results}

Table 1 shows the outcomes of the FASTPlaque-Response test, with and without NOA, compared with culture and the conventional DST method. When the three methods were compared for specimens up to 3 days old, the percentage of interpretable results was significantly higher in the FASTPlaque-Response test with NOA (87.8\%) compared with the FASTPlaque-Response test without
NOA $(69.3 \%)$ and culture-based DST $(70.1 \%)(P<0.001)$. There was no significant difference in the proportion of interpretable results between the FASTPlaque-Response test without NOA and culture-based DST $(P=0.849)$.

This improvement in the interpretability of results for the FASTPlaque-Response test with NOA is likely to enhance the cost-effectiveness of rapid rifampicin testing, as fewer repeat tests will be required. Furthermore, providing more results quickly is likely to reduce the number of patients not receiving their results because they fail to return for follow-up appointments. For specimens of 4 days or older, the proportion of interpretable results was slightly higher for the conventional method $(76.0 \%)$ than for the FASTPlaque-Response with NOA $(73.7 \%)$ and higher than that for FASTPlaque-Response without NOA (69.3\%). When all specimens were considered, FASTPlaqueResponse had the highest proportion of interpretable results (79.0\%), followed by conventional DST (73.8\%) and then FASTPlaque-Response without NOA (69.3\%).

Effective control of contamination in the FASTPlaqueResponse test with NOA $(0.8 \%$ contamination rate in specimens aged $\leqslant 3$ days) was a significant factor in the higher level of interpretable results observed. A low level of invalid results (RIF- $<100$ plaques) observed with NOA (6.7\%) compared with without NOA (15.5\%) was also a contributing factor. The reason for the reduced number of

Table 1. Comparison of FASTPlaque-Response results, with and without NOA, with conventional DST

\begin{tabular}{|c|c|c|c|}
\hline & \multicolumn{3}{|c|}{ No. (\%) of interpretable results from: } \\
\hline & $\begin{array}{c}\text { FASTPlaque-Response } \\
\text { with NOA }\end{array}$ & $\begin{array}{c}\text { FASTPlaque-Response } \\
\text { without NOA }\end{array}$ & $\begin{array}{c}\text { Culture }+ \\
\text { conventional DST }\end{array}$ \\
\hline \multicolumn{4}{|l|}{$\begin{array}{l}\text { Specimens aged } \leqslant 3 \text { days } \\
(n=238)\end{array}$} \\
\hline Total interpretable results & $209(87.8)$ & $165(69.3)$ & $167(70.1)$ \\
\hline Contamination & $2(0.8)$ & $34(14.3)$ & $61(25.6)$ \\
\hline Invalid RIF- & $16(6.7)$ & $38(16)$ & NA \\
\hline $\begin{array}{l}\text { Controls out of spec/results not } \\
\text { reported }\end{array}$ & $11(4.6)$ & $1(0.4)$ & $10(4.2)^{*}$ \\
\hline \multicolumn{4}{|l|}{ Specimens $\geqslant 4$ days $(n=391)$} \\
\hline Total interpretable results & $288(73.7)$ & $271(69.3)$ & $297(76.0)$ \\
\hline Contamination & $24(6.1)$ & $54(13.8)$ & $66(16.9)$ \\
\hline Invalid RIF- & $69(17.6)$ & $57(14.6)$ & NA \\
\hline $\begin{array}{l}\text { Controls out of spec/results not } \\
\text { reported }\end{array}$ & $10(2.6)$ & $9(2.3)$ & $28(7.2)$ \\
\hline \multicolumn{4}{|l|}{ All specimens $(n=629)$} \\
\hline Total interpretable results & $497(79.0)$ & $436(69.3)$ & $464(73.8)$ \\
\hline Contamination & $26(4.1)$ & $88(13.9)$ & $127(20.2)$ \\
\hline Invalid RIF- & $85(13.5)$ & $95(15.1)$ & NA \\
\hline $\begin{array}{l}\text { Controls out of spec/results not } \\
\text { reported }\end{array}$ & $21(3.3)$ & $10(1.6)$ & $38(6.0)^{*}$ \\
\hline
\end{tabular}

NA, Not applicable.

${ }^{\star}$ Result not reported for culture due to either a negative culture, the presence of non-tuberculous mycobacteria or loss of the specimen in the reporting system. 
invalid results with NOA was unknown and unexpected, as the presence of antimicrobials in the medium was not anticipated to improve the viability or detection of $M$. tuberculosis in the specimens. However, when all specimens were considered, this effect on invalid results was less pronounced and was in keeping with previous reports (Albert et al., 2002)

Contamination rates for MGIT culture followed by DST on 7H11 solid medium were higher than the rate seen with FASTPlaque-Response, with $20.2 \%$ of specimens lost due to contamination. Specimens were lost primarily due to contamination of the primary MGIT culture, which occurred at a higher rate than reported elsewhere $(8.6 \%$, Cruciani et al., 2004), despite the fact that PANTA antibiotic supplement was used in the primary MGIT culture, as recommended by the manufacturer. Many of the contaminated MGIT cultures were mixed cultures, containing both acid-fast and non-acid-fast bacteria. Additional decontamination of these mixed cultures is likely to increase the number of interpretable results from culture, but would have resulted in increased workload and longer reporting times. This approach was not routinely used in the NHLS laboratory due to very high throughput. This high contamination rate is unlikely to be due to the age of the specimen giving rise to the opportunity for contaminating organisms to grow prior to decontamination, as less contamination was observed overall $(20.2 \%)$ compared with specimens aged $\leqslant 3$ days $(25.6 \%)$. The majority of specimens $(57.4 \%)$ were either 2 or 4 days old. There was a significant difference $(P=0.0251)$ in contamination between 2- and 4-day-old specimens, with lower contamination in cultures from older specimens.

In the FASTPlaque-Response test, contamination can overgrow the results plates and prevent visualization of plaques. The FASTPlaque-Response test had less contamination than the culture-based DST in this study, even in the absence of NOA ( $13.9 \%$ compared with $20.2 \%$ ). However, the presence of NOA reduced contamination further (to $4.1 \%$ ), permitting more results to be interpreted. The NOA antimicrobial was effective in reducing contamination to acceptable limits.

Invalid results in which there were inadequate plaques in the drug-free plate were the other main cause of loss of interpretability of results in the FASTPlaque-Response assay. A drug-free control plate without rifampicin (RIF-), set up for each specimen, was required to produce at least 100 plaques for interpretation of the RIF + plate to be valid. Although only smear-positive specimens were included in the study, where large numbers of $M$. tuberculosis cells are expected in each specimen, it is known from previous studies that approximately 10-15\% of smear-positive specimens do not support productive phage infection (Albert et al., 2002, 2004; Muzaffar et al., 2002). This is unlikely to be due to the host range of the bacteriophage, as most of the isolated $M$. tuberculosis strains from these specimens can subsequently be phage-infected from the primary culture (Albert et al.,
2002). Instead, this is likely to be due to the harsh decontamination procedure damaging the $M$. tuberculosis cells and reducing their ability to support productive phage infection, or from the suggested presence of phage inhibitors in sputum (Albert et al., 2002, 2004; Muzaffar et al., 2002). The level of invalid RIF- results for all specimens appeared to be consistent with these earlier findings.

There were some specimens where the batch control result was invalid (negative control $\geqslant 10$ ). A single batch of specimens in each of the FASTPlaque-Response test procedures (on different days) failed and was responsible for this loss of results. The most likely explanation for this effect was operator error.

The beneficial effects of NOA were diminished somewhat when older specimens were included in the analysis (Table 1). The interpretability of the FASTPlaqueResponse test with NOA declined from $87.8 \%$ with fresher specimens to $79.0 \%$ in the overall analysis due to a larger proportion of invalid results $(13.5 \%)$ and contaminated results $(4.1 \%)$. The overall frequency of invalid results was similar to that seen without NOA $(15.1 \%)$ and to other studies (Albert et al., 2002, 2004; Muzaffar et al., 2002). Contamination was higher than seen in fresher specimens. However, this may be an overestimation of the specimenrelated contamination, as $21 / 26$ specimens that were contaminated came from a single day's batch of results and all of the contaminants had similar colony morphology, suggesting that the contaminant had been introduced into the culture medium due to operator error.

\section{FASTPlaque-Response performance}

The performance of the FASTPlaque-Response test, with and without NOA, was compared with conventional DST, with specimens aged $\leqslant 3$ days (Table 2 ) and aged $\geqslant 4$ days (Table 3) where valid results for the FASTPlaque-Response and conventional DST methods were available. The performance of the FASTPlaque-Response test was similar in the presence or absence of NOA (Table 4). In addition, the performance of the phage-based test compared with conventional DST was similar to other studies (Albert et al., 2004; Guerra et al., 2005). Neither the age of the specimen ( $\leqslant 3$ days old compared with $\geqslant 4$ days) nor the smear grading $(1+, 2+$ or $3+)$ had a substantial effect on the ability of the FASTPlaque-Response test to identify rifampicin resistance correctly (data not shown).

Samples with discrepant results were resolved through repeat conventional testing and $r p o B$ mutation analysis. In all but 2 cases (of 15 evaluated), the rpoB result agreed with the culture-based DST result. In these two strains that were susceptible by conventional DST but resistant by the FASTPlaque-Response method, both were found to have mutations at position 511 of the rpoB gene and were therefore considered to be resistant for the resolved data analysis. Performance data using the resolved DST results are presented in Table 4 . 
Table 2. Comparison of FASTPlaque-Response results (with and without NOA) with conventional DST results for specimens aged $\leqslant 3$ days (resolved data in parentheses)

\begin{tabular}{|clcc|}
\hline & & \multicolumn{2}{c|}{$\begin{array}{c}\text { Conventional } \\
\text { culture DST }\end{array}$} \\
\cline { 2 - 4 } & & Resistant & Susceptible \\
\hline $\begin{array}{c}\text { FASTPlaque-Response } \\
\text { with NOA }\end{array}$ & Resistant & $21(22)$ & $6(5)$ \\
$\begin{array}{c}\text { FASTPlaque-Response } \\
\text { without NOA }\end{array}$ & $\begin{array}{l}\text { Susceptible } \\
\text { Resistant }\end{array}$ & $0(0)$ & $126(126)$ \\
& Susceptible & $0(0)$ & $97(5)$ \\
\hline
\end{tabular}

\section{Potential applications of the FASTPlaque- Response method}

These data demonstrate that the NOA antimicrobial supplement may be used with the FASTPlaque-Response test to reduce the number of results lost due to contamination, without any detriment to the performance of the test. Reducing the number of uninterpretable results will result in better service provision, more reliable and rapid results, and improved cost-effectiveness. Use of the NOA supplement with the FASTPlaqueResponse test resulted in approximately $87 \%$ of specimens (aged $\leqslant 3$ days) yielding results, which was a higher level of available results than conventional DST in this study. The FASTPlaque-Response test gave results comparable to the conventional DST method, with overall accuracy, sensitivity and specificity all greater than $95 \%$. However, the FASTPlaque-Response test yielded results in 2 days (depending on the working system of the laboratory and whether laboratory staff worked over the weekends) compared with the slower conventional DST result which took an average time of 35.3 days (range 26-77 days).
Table 3. Comparison of FASTPlaque-Response results (with and without NOA) with conventional DST results for specimens aged $\geqslant 4$ days (resolved data in parentheses)

\begin{tabular}{|c|c|c|c|}
\hline & & \multicolumn{2}{|c|}{$\begin{array}{c}\text { Conventional culture } \\
\text { DST }\end{array}$} \\
\hline & & Resistant & Susceptible \\
\hline \multirow{2}{*}{$\begin{array}{l}\text { FASTPlaque-Response } \\
\text { with NOA }\end{array}$} & Resistant & $30(31)$ & $8(7)$ \\
\hline & Susceptible & $1(1)$ & 191 (191) \\
\hline \multirow{2}{*}{$\begin{array}{l}\text { FASTPlaque-Response } \\
\text { without NOA }\end{array}$} & Resistant & $21(22)$ & $7(6)$ \\
\hline & Susceptible & $3(3)$ & $190(190)$ \\
\hline
\end{tabular}

In addition, these data suggest that the test may be used with older specimens ( $\geqslant 4$ days) with comparable levels of performance to conventional DST. However, slightly reduced levels of interpretation may be encountered compared with fresher specimens. Where there is slow transport from the collection site to the laboratory, or in low-throughput laboratories where batching of specimens is required, the ability to test older specimens may be advantageous.

Application of the FASTPlaque-Response method for the screening of $\mathrm{TB}$ patients will be influenced by the performance of the test, the incidence of rifampicin resistance in the population and the correlation of rifampicin resistance with MDR TB. The type of patient suitable for testing may vary in different settings depending on the incidence of MDR TB and the correlation of rifampicin resistance with MDR TB. A presumptive diagnosis of MDR TB could be made based on detection of rifampicin resistance by the FASTPlaque-Response test within 2 days. Further DST could be carried out to confirm the result and for testing susceptibility to additional drugs for individualized MDR therapy, where appropriate.

Table 4. Resolved performance parameters of the FASTPlaque-Response test result (with or without NOA) compared with conventional DST

The $95 \%$ confidence interval is shown in parentheses. PPV, Positive predictive value; NPV, negative predictive value.

\begin{tabular}{|c|c|c|c|c|c|c|}
\hline \multirow[t]{2}{*}{ Result } & \multicolumn{3}{|c|}{ With NOA } & \multicolumn{3}{|c|}{ Without NOA } \\
\hline & $\leqslant 3$ days old & $\geqslant 4$ days old & All specimens & $\leqslant 3$ days old & $\geqslant 4$ days old & All specimens \\
\hline Overall agreement & 96.7 (93.9-99.5) & $96.5(94.1-98.9)$ & $96.6(94.8-98.4)$ & $95.9(92.4-99.4)$ & $95.9(93.3-98.5)$ & $95.9(93.8-98.0)$ \\
\hline Sensitivity & $100.0(83.9-100.0)$ & $96.9(90.9-100.0)$ & $98.1(94.5-100.0)$ & $100.0(81.5-100.0)$ & $88(75.3-100.0)$ & $93.2(85.8-100.0)$ \\
\hline Specificity & $96.2(91.9-99.5)$ & $96.5(93.9-99.1)$ & $96.3(94.3-98.3)$ & $95.1(90.9-99.3)$ & $96.9(94.5-99.3)$ & $96.3(94.2-98.4)$ \\
\hline PPV & $81.5(66.9-96.1)$ & $81.6(69.3-93.9)$ & $81.5(72.1-90.9)$ & $79.2(63.0-95.4)$ & $78.6(63.4-93.8)$ & $78.8(67.7-89.9)$ \\
\hline NPV & $100.0(97.1-100.0)$ & $99.5(97.1-100.0)$ & $99.7(99.1-100.0)$ & $100.0(96.3-100.0)$ & $98.5(96.8-100.0)$ & $99.0(97.9-100.0)$ \\
\hline
\end{tabular}




\section{ACKNOWLEDGEMENTS}

The authors would like to thank the staff of the NHLS in Greenpoint, Cape Town, for their help, and Professor Tommie Victor, University of Stellenbosch, South Africa, for performing the $r p o B$ sequence analysis of the discrepant strains. The Foundation for Innovative New Diagnostics (FIND), a Geneva-based non-profit foundation focused on new TB diagnostic technologies for use in high burden countries, has a co-development agreement with Biotec Laboratories Ltd, which supports the development, evaluation and demonstration of the FASTPlaque-Response test. FIND is currently evaluating the test for public sector use. The authors are grateful to Dr Mark Perkins and Dr Rick O'Brien for reviewing the manuscript.

\section{REFERENCES}

Albert, H., Heydenrych, A., Mole, R., Trollip, A. P. \& Blumberg, L. (2001). Evaluation of FASTPlaqueTB-RIF, a rapid, manual test for the determination of rifampicin resistance from $M$. tuberculosis cultures. Int J Tuberc Lung Dis 5, 906-911.

Albert, H., Heydenrych, A., Brookes, R., Mole, R. J., Harley, B., Subotsky, E., Henry, R. \& Azevedo, V. (2002). Performance of a rapid phage-based test, FASTPlaqueTB, to diagnose pulmonary tuberculosis from sputum specimens in South Africa. Int J Tuberc Lung Dis 6, 529-537.

Albert, H., Trollip, A., Seaman, T. \& Mole, R. J. (2004). Simple, phagebased (FASTPlaque) technology to determine rifampicin resistance of Mycobacterium tuberculosis directly from sputum. Int J Tuberc Lung Dis 8, 1114-1119.

Allen, B. W. (1984). Tuberculosis bacteriology in developing countries. Med Lab Sci 41, 400-409.

Cruciani, M., Scarparo, C., Malena, M., Bosco, O., Serpelloni, G. \& Mengoli, C. (2004). Meta-analysis of BACTEC MGIT 960 and BACTEC 460TB, with and without solid media, for detection of Mycobacteria. J Clin Microbiol 42, 2321-2325.

Enarson, D. A., Rieder, H. L., Arnadottir, T. \& Trebucq, A. (2000). Management of Tuberculosis. A Guide for Low Income Countries. Paris: International Union Against Tuberculosis and Lung Disease.

Fischl, M. A., Daikos, G. L., Uttamchandrani, R. B., Poblete, R. B., Moreno, J. N., Reyes, R. R., Boota, A. M., Thompson, L. M., Cleary, T. J. \& other authors (1992). Clinical presentation and outcome of patients with HIV infection and tuberculosis caused by multiple drug resistant bacilli. Ann Intern Med 117, 184-190.

Guerra, H., Henostroza, G., Rojas, G. \& other authors (2005). Evaluation of a phage amplification assay for detection of MDR-TB in smear-positive patients in Lima, Peru. Int J Tuberc Lung Dis 9 (Suppl. 1), S196.

Heifets, L. B. \& Cangelosi, G. A. (1999). Drug susceptibility testing in Mycobacterium tuberculosis: a neglected problem at the turn of the century. Int J Tuberc Lung Dis 3, 564-581.
Jacobs, W. R., Barletta, R. G., Udani, R., Chan, J., Kalkut, G., Sosne, G., Kieser, T., Sarkis, G. J., Hatfull, G. F. \& Bloom, B. R. (1993). Rapid assessment of drug susceptibilities of Mycobacterium tuberculosis by means of luciferase reporter phages. Science 260, 819-822.

Kent, P. T. \& Kubica, G. P. (1985). Public Health Mycobacteriology. A Guide for the Level III Laboratory. Atlanta, GA: Centres for Disease Control.

Kisa, O., Albay, A., Bedir, O., Baylan, O. \& Doganci, L. (2003). Evaluation of FASTPlaqueTB-RIF for determination of rifampicin resistance in Mycobacterium tuberculosis complex isolates. Int J Tuberc Lung Dis 7, 284-288.

Morgan, M., Kalantri, S., Flores, L. \& Pai, M. (2005). A commercial line probe assay for the rapid detection of rifampicin resistance in Mycobacterium tuberculosis: a systematic review and meta-analysis. BMC Infect Dis 5, 62.

Muzaffar, R., Batool, S., Aziz, F., Naqvi, A. \& Rizvi, A. (2002). Evaluation of the FASTPlaqueTB assay for direct detection of Mycobacterium tuberculosis in sputum specimens. Int J Tuberc Lung Dis 6, 635-640.

Pai, M., Kalantri, S. \& Dheda, K. (2006). New tools and emerging technologies for the diagnosis of tuberculosis: part II. Active tuberculosis and drug resistance. Expert Rev Mol Diagn 6, 423-432.

Seaman, T., Trollip, A., Mole, R. \& Albert, H. (2003). The use of a novel phage-based technology as a practical tool for the diagnosis of tuberculosis in Africa. Afr J Biotechnol 2, 40-45.

Suarez, P. G., Floyd, K., Portocarrero, J., Alarcon, E., Rapiti, E., Ramos, G., Bonilla, C., Sabogal, I., Aranda, I. \& other authors (2002). Feasibility and cost-effectiveness of standardised second-line drug treatment for chronic tuberculosis patients: a national cohort study in Peru. Lancet 359, 1980-1989.

Victor, T. C., Jordaan, A. M., van Rie, A., van der Spuy, G. D., Richardson, M., van Helden, P. D. \& Warren, R. (1999). Detection of mutations in drug resistance genes of Mycobacterium tuberculosis by a dot-blot hybridisation strategy. Tuber Lung Dis 79, 343-348.

WHO (2003). Treatment of Tuberculosis: Guidelines for National Programs. Geneva: World Health Organization.

WHO (2004). Anti-Tuberculosis Drug Resistance in the World: Report 3. Geneva: World Health Organization.

Wilson, S. M. (1997). Method to detect bacteria. PCT patent WO97/ 022713.

Wilson, S. M., Al-Suwaidi, Z., McNerney, R., Porter, J. \& Drobniewski, F. (1997). Evaluation of a new rapid bacteriophage-based method for the drug susceptibility testing of Mycobacterium tuberculosis. Nat Med 3, $465-468$.

Zignol, M., Hosseini, M. S., Wright, A., Weezenbeek, C. L., Nunn, P., Watt, C. J., Williams, B. G. \& Dye, C. (2006). Global incidence of multidrug-resistant tuberculosis. J Infect Dis 194, 479-485. 